\title{
Description of a Hospital Outbreak of Middle East Respiratory Syndrome in a Large Tertiary Care Hospital in Saudi Arabia
}

\author{
Hanan H. Balkhy, MD; ${ }^{1,2,3}$ Thamer H. Alenazi, MD; ${ }^{1,3}$ Majid M. Alshamrani, MD; ${ }^{4}$ Henry Baffoe-Bonnie, MD; ${ }^{1,3}$ \\ Yaseen Arabi, MD; ${ }^{1,5}$ Raed Hijazi, MD; ${ }^{1,6}$ Hail M. Al-Abdely, MD; ${ }^{7}$ Aiman El-Saed, MD, PhD; ${ }^{1,3}$ Sameera Al Johani, MD; ${ }^{8}$ \\ Abdullah M. Assiri, MD; ${ }^{7,9}$ Abdulaziz bin Saeed, MD, $\mathrm{PhD}^{7,10}$
}

\begin{abstract}
BACKGROUnd. Since the first isolation of Middle East respiratory syndrome coronavirus (MERS-CoV) in Saudi Arabia in 2012, sporadic cases, clusters, and sometimes large outbreaks have been reported.

oвjective. To describe the recent (2015) MERS-CoV outbreak at a large tertiary care hospital in Riyadh, Saudi Arabia.

метнорs. We conducted an epidemiologic outbreak investigation, including case finding and contact tracing and screening. MERS-CoV cases were categorized as suspected, probable, and confirmed. A confirmed case was defined as positive reverse transcription polymerase chain reaction test for MERS-CoV.

RESUlts. Of the 130 suspected cases, $81(62 \%)$ were confirmed and $49(38 \%)$ were probable. These included 87 patients $(67 \%)$ and 43 healthcare workers (33\%). Older age (mean [SD], 64.4 [18.3] vs 40.1 [11.3] years, $P<.001$ ), symptoms (97\% vs $58 \%, P<.001$ ), and comorbidity $(99 \%$ vs $42 \%, P<.001)$ were more common in patients than healthcare workers. Almost all patients $(97 \%)$ were hospitalized whereas most healthcare workers (72\%) were home isolated. Among 96 hospitalized cases, 63 (66\%) required intensive care unit management and 60 (63\%) required mechanical ventilation. Among all 130 cases, 51 (39\%) died; all were patients (51 [59\%]) with no deaths among healthcare workers. More than half (54\%) of infections were believed to be caught at the emergency department. Strict infection control measures, including isolation and closure of the emergency department, were implemented to interrupt the chain of transmission and end the outbreak.
\end{abstract}

CONCLUSION. MERS-CoV remains a major healthcare threat. Early recognition of cases and rapid implementation of infection control measures are necessary.

Infect Control Hosp Epidemiol 2016;37:1147-1155

Since the first diagnosis of Middle East respiratory syndrome (MERS), caused by the MERS coronavirus (MERS-CoV), in 2012 in Saudi Arabia, ${ }^{1}$ 1,283 laboratory-confirmed cases of MERS-CoV have been reported in Saudi Arabia as of December 31,2015 , including at least 551 related deaths with approximate case fatality of $43 \%{ }^{2}$ This represented approximately $80 \%$ of the total number of globally reported cases of MERS-CoV. ${ }^{3}$ According to the Saudi Ministry of Health, $45 \%$ of the reported cases were classified as healthcare-acquired infections among either patients or healthcare workers (HCWs). ${ }^{2}$ Although sporadic cases have been reported in Saudi hospitals during the past 3 years, MERS-CoV infections in healthcare settings are frequently seen as small clusters or sometimes large outbreaks. ${ }^{4}$ For example, small clusters including a total of 23 cases have been detected in 3 healthcare facilities in the Eastern region between
April and May 2013. ${ }^{5}$ Additionally, a large hospital outbreak with 255 cases has been reported from multiple hospitals in Jeddah in the first half of 2014 with the peak between March and April $2014 .{ }^{6}$ The recent Korean outbreak that involved 186 MERS-CoV cases, including 36 deaths, has further raised the global concern of the possibility of large hospital outbreaks even with only one or a few community cases. ${ }^{7,8}$

In June 2013, the first case of MERS-CoV infection was confirmed at our hospital, King Abdulaziz Medical City, Riyadh (KAMC-R). Since then, a total of 32 patients and 14 HCWs have had confirmed MERS-CoV infections through early March 2015. ${ }^{9}$ The cases were distributed as sporadic cases and small clusters. In late June 2015 (3.5 months from the last recognized MERS-CoV case in our hospital), the emergency department (ED) evaluated the first case of the current

Affiliations: 1. King Saud bin Abdulaziz University for Health Sciences, Riyadh Saudi Arabia; 2. King Abdullah International Medical Research Center; 3. Infection Prevention and Control Department, King Abdulaziz Medical City, Riyadh, Saudi Arabia; 4. Infection Prevention and Control Department, King Abdulaziz Medical City, Jeddah, Saudi Arabia; 5. Intensive Care Department, King Abdulaziz Medical City, Riyadh, Saudi Arabia; 6. Emergency Care Department, King Abdulaziz Medical City, Riyadh, Saudi Arabia; 7. Ministry of Health, Riyadh, Saudi Arabia; 8. Pathology and Laboratory Medicine Department, King Abdulaziz Medical City, Riyadh, Saudi Arabia; 9. King Fahad Medical City, Ministry of Health, Riyadh, Saudi Arabia; 10. King Saud University, Riyadh, Saudi Arabia.

Received February 11, 2016; accepted April 18, 2016; electronically published July 18, 2016

(c) 2016 by The Society for Healthcare Epidemiology of America. All rights reserved. 0899-823X/2016/3710-0003. DOI: 10.1017/ice.2016.132 
outbreak, which was ongoing through early September 2015. The objective of the current study was to describe the 2015 KAMC-R outbreak, with special attention to the differences in the course and outcomes between patients and HCWs.

\section{METHODS}

\section{Setting}

The current study was conducted at KAMC-R, which is an approximately 1,000-bed tertiary care facility. The ED is a 150-bed multisection complex area that serves more than 250,000 visits a year. There are 13 different intensive care units (ICUs) in the institution with a bed capacity of 185 serving approximately 3,000 admissions and 57,000 patient-days of care every year. Additionally, other specialties are covered in 36 wards serving approximately 27,000 admissions and 244,000 patient-days of care every year. KAMC-R provides healthcare services to approximately 750,000 Saudi National Guard soldiers, employees, and their families. The care provided ranges from primary and preventive care to tertiary care. Approximately 9,170 HCWs were working for KAMC-R in jobs that involve direct patient care, including approximately 1,670 physicians, 4,660 nurses, and 2,840 other HCWs. Additionally, another 3,400 individuals were working for KAMC-R in administrative jobs that involve no direct patient care. The data about the served population and HCWs of KAMC-R were obtained from the annual census reports for 2013 and 2014.

\section{Study Design}

The current study design was a prospective surveillance study for all suspected patients and exposed HCWs at KAMC-R during the outbreak period, mid-June to mid-September, 2015.

\section{Case Definition}

The population screened in the current study was suspected patients either admitted or evaluated at the ED as well as exposed HCWs. The case definition used was based on the definition update released by the Saudi Ministry of Health in June 2015. ${ }^{10}$ Suspected patients were defined as those who had fever and/or respiratory symptoms or received a diagnosis of pneumonia (community-acquired or healthcare-acquired) based on clinical or radiologic evidence, with or without documented exposure to camels or to a confirmed/probable case of MERS-CoV infection in the previous 2 weeks. Exposed HCWs were defined as those who had been working in any department of KAMC-R during the outbreak period (mid-June to mid-September, 2015) and who had direct exposure (within 2 meters) or indirect exposure (being in the same room/department) to a confirmed or probable case of MERS-CoV infection within the previous 2 weeks with or without symptoms. A probable case was defined as a suspected patient or exposed HCW with inconclusive laboratory results (such as lack of confirmation by the Ministry of Health central laboratory, or having positive polymerase chain reaction on only 1 of the 2 genomic targets). A confirmed case was defined as a suspected patient or exposed HCW who had a positive reverse transcriptionpolymerase chain reaction test for MERS-CoV (Real-Time RT-PCR Kit; TibMolbiol). The sensitivity and specificity of the test were determined locally at $96 \%$ and $95 \%$, respectively.

\section{Case Finding}

Once a case was classified as probable or confirmed, a list of all exposed HCWs and/or patients was generated (by respective department and/or HCWs) and contact tracing was started. Nasopharyngeal swab samples were obtained from all exposed HCWs and nasopharyngeal swab samples or other appropriate types of specimens were obtained from all exposed/suspected patients. Once a case was classified as probable or confirmed, infection preventionists collected demographic information, exposure, medical history, symptoms, and outcome.

\section{Control Measures}

As the first 2 cases were admitted on June 21 and July 15, respectively, the preexisting Infectious Disease Epidemic Plan, which was established by the local hospital outbreak committee on the basis of US Centers for Disease Control and Prevention ${ }^{11}$ and World Health Organization ${ }^{12}$ guidelines, was activated during the week of August 2. The plan included strict enforcing of infection control measures, including droplet/ contact isolation for suspected and probable cases and airborne/contact isolation for confirmed cases in addition to strict hand hygiene compliance. As the situation escalated, stricter measures were implemented, including airborne/ contact isolation for suspected/probable cases. Because of ongoing identification of new cases, the plan was escalated on August 18, 2015, to the highest level-that is, level 3, which includes closure of the ED, cancellation of elective surgical procedures, and suspension of all outpatient appointments and visits. Complete evacuation of the ED was achieved on August 22,2015 . With shutdown of the hospital services and engineering modifications, more locations (ICUs and wards) were dedicated for isolation and more rooms were converted to negative pressure rooms. Probable or confirmed HCWs were allowed to return to work after having a symptom-free week in addition to at least 1 negative reverse transcription-polymerase chain reaction test. The end of the outbreak was declared on September 28, 2015, after the completion of two 14-day incubation periods without further identification of new cases.

\section{Statistical Methods}

The number of MERS-CoV cases was plotted against weeks of onset of symptoms between mid-June and mid-September, 2015 , to create the epidemic curve. For asymptomatic cases, the date of onset was replaced by the date of sample collection. Series of epidemic curve were further stratified by the type of 
case, diagnostic classification, symptoms, location of isolation, and different outcome measures. Because the screening and the behavior of the MERS-CoV infection was considerably different in patients and HCWs, we compared demographic information, symptoms, medical history, and outcome measures between both groups. The $\chi^{2}$ or Fisher exact test, as appropriate, was used to test significant differences of categorical variables between the 2 study groups. The $t$ test or Mann-Whitney test, as appropriate, was used to test significant differences of continuous variables between the 2 study groups. All $P$ values were 2 -tailed and $P<.05$ was considered significant. SPSS, version 22.0 (IBM), was used for all statistical analyses.

\section{RESULT S}

During the outbreak period, a total of 130 cases, including 87 patients (67\%) and $43 \mathrm{HCWs}(33 \%)$, were diagnosed as confirmed $(n=81)$ or probable $(n=49)$ MERS-CoV infection. The demographic characteristics and classification of cases are shown in Table 1. The mean (SD) age of cases was 64.4 (18.3)

TAвLE 1. Demographic Characteristics and Classification of MERS-CoV Cases at KAMC-Riyadh (Mid-June to Mid-September, 2015)

\begin{tabular}{|c|c|c|c|c|}
\hline Variable & Patients $(\mathrm{N}=87)$ & HCWs $(\mathrm{N}=43)$ & Overall $(\mathrm{N}=130)$ & $P$ value \\
\hline \multicolumn{5}{|l|}{ Age } \\
\hline Mean (SD) & $64.4(18.3)$ & $40.1(11.3)$ & $56.3(19.9)$ & $<.001$ \\
\hline$<40$ years & $9(10 \%)$ & $24(56 \%)$ & $33(25 \%)$ & $<.001$ \\
\hline $40-60$ years & $26(30 \%)$ & $18(42 \%)$ & $44(34 \%)$ & \\
\hline$>60$ years & $52(60 \%)$ & $1(2 \%)$ & $53(41 \%)$ & \\
\hline \multicolumn{5}{|l|}{ Gender } \\
\hline Male & $56(64 \%)$ & $10(23 \%)$ & $66(51 \%)$ & $<.001$ \\
\hline Female & $31(36 \%)$ & $33(77 \%)$ & $64(49 \%)$ & \\
\hline \multicolumn{5}{|l|}{ BMI group } \\
\hline Mean (SD) & $28.8(6.7)$ & $26.3(4.2)$ & $28.1(6.2)$ & .03 \\
\hline Normal & $28(33 \%)$ & $15(39 \%)$ & $43(35 \%)$ & .44 \\
\hline Overweight & $27(32 \%)$ & $14(37 \%)$ & $41(33 \%)$ & \\
\hline Obese & $30(35 \%)$ & $9(24 \%)$ & $39(32 \%)$ & \\
\hline \multicolumn{5}{|l|}{ Nationality } \\
\hline Saudi & $82(94 \%)$ & $7(16 \%)$ & $89(68 \%)$ & $<.001$ \\
\hline Non-Saudi & $5(6 \%)$ & $36(84 \%)$ & $41(32 \%)$ & \\
\hline Philippines & $0(0 \%)$ & $32(74 \%)$ & $32(25 \%)$ & $<.001$ \\
\hline Middle East & $5(6 \%)$ & $0(0 \%)$ & $5(4 \%)$ & \\
\hline Others & $0(0 \%)$ & $4(9 \%)$ & $4(3 \%)$ & \\
\hline Eligibility of care at NGHA & & & & $<.001$ \\
\hline No & $31(36 \%)$ & $0(0 \%)$ & $31(24 \%)$ & \\
\hline Yes, employee & $20(23 \%)$ & $43(100 \%)$ & $63(48 \%)$ & \\
\hline Yes, dependent & $36(41 \%)$ & $0(0 \%)$ & $36(28 \%)$ & \\
\hline \multicolumn{5}{|c|}{ Professional category of HCWs } \\
\hline Nurse & & $27(63 \%)$ & $27(63 \%)$ & NA \\
\hline Physician & & $5(12 \%)$ & $5(12 \%)$ & \\
\hline Other HCWs & & $11(26 \%)$ & $11(26 \%)$ & \\
\hline \multicolumn{5}{|l|}{ Department of HCWs } \\
\hline Emergency department & & $20(47 \%)$ & $20(47 \%)$ & NA \\
\hline Wards & & $10(23 \%)$ & $10(23 \%)$ & \\
\hline Outpatient clinics & & $4(9 \%)$ & $4(9 \%)$ & \\
\hline Intensive care units & & $4(9 \%)$ & $4(9 \%)$ & \\
\hline Multiple locations & & $5(12 \%)$ & $5(12 \%)$ & \\
\hline \multicolumn{5}{|l|}{ Diagnostic classification } \\
\hline Confirmed & $67(77 \%)$ & $14(33 \%)$ & $81(62 \%)$ & $<.001$ \\
\hline Probable & $20(23 \%)$ & $29(67 \%)$ & $49(38 \%)$ & \\
\hline \multicolumn{5}{|l|}{ Type of transmission } \\
\hline Community-acquired & $29(33 \%)$ & $0(0 \%)$ & $29(22 \%)$ & $<.001$ \\
\hline Healthcare-acquired & $58(67 \%)$ & $43(100 \%)$ & $101(78 \%)$ & \\
\hline
\end{tabular}

NOTE. The column heads represent the maximum number; the denominator was sometimes lower when data were missing. BMI, body mass index (calculated as weight in kilograms divided by height in meters squared); HCW, healthcare worker; KAMC, King Abdulaziz Medical City; MERS-CoV, Middle East respiratory syndrome coronavirus; NGHA, National Guard Health Affairs. 
years, with patients having significantly older age than HCWs (56.3 [19.9] vs 40.1 [11.3] years, $P<.001$ ). Overall, males and females were equally represented but males represented the majority $(64 \%)$ of patients whereas females represented the majority $(77 \%)$ of HCWs $(P<.001)$. The majority $(94 \%)$ of patients were Saudi whereas the majority $(84 \%)$ of HCWs were non-Saudi, mainly from the Philippines $(P<.001)$. Patients had slightly higher body mass index (calculated as weight in kilograms divided by height in meters squared) than HCWs (28.8 [6.7] vs 26.3 [4.2], $P=.03$ ), with no significant difference in the percentage of obesity between the 2 groups. The patients were either National Guard Health Affairs employees (23\%) or their dependents (41\%), and approximately one-third (36\%) of the patients were not from the official National Guard Health Affairs served population. The majority (63\%) of HCWs were nurses, and the rest were either physicians (12\%) or other HCWs (26\%), mainly patient care technicians and respiratory therapists. The majority $(47 \%)$ of HCWs were working at the ED, while the rest were working in wards $(23 \%)$, outpatient clinics (9\%), ICUs (9\%), and multiple locations within the KAMC-R (12\%). The majority $(86 \%)$ of HCWs were discovered after screening of exposed HCWs, with $37(1.2 \%)$ positive of 2,992 processed swabs.

The majority (77\%) of patients were classified as confirmed cases whereas the majority (67\%) of HCWs were classified as probable $(P<.001)$. Whereas all HCW infections $(n=43)$ were classified as healthcare-acquired, one-third $(n=29)$ of patients had community-acquired infection and two-thirds $(\mathrm{n}=58)$ had healthcare-acquired infection. As shown in Figure 1A, the community-acquired infections started the outbreak and continued through the peak. On the other hand, healthcare-acquired infections were the majority of cases during the center of the outbreak and the only type of transmission toward its end. Of the healthcare-acquired infections, more than half (54\%) were believed to be caught at the ED and

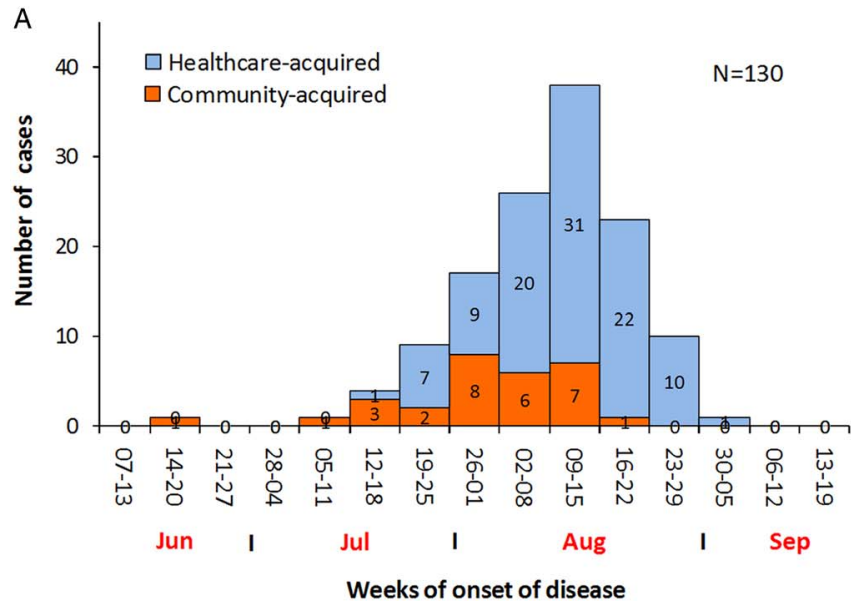

$24 \%$ were caught at wards. Although decreasing during the second half of the outbreak, transmission at the ED occurred throughout the outbreak (Fig. 1B).

As shown in Table 2, 84\% of all cases were symptomatic, with higher frequency of symptomatic cases among patients than HCWs $(97 \%$ vs $58 \%, P<.001)$. Additionally, among symptomatic cases, patient reported higher mean (SD) number of all symptoms (5.4 [2.4] vs $4.2[2.3], P<.03)$ but similar number of respiratory symptoms (2.6 [1.4] vs 2.4 [1.3], $P=.54$ ) compared with HCWs. Eighteen of the 21 asymptomatic cases (detected during contact screening) were HCWs. As the frequency of HCWs relatively increased towards the end of the outbreak (Fig. 2A), the frequency of probable diagnosis (and asymptomatic cases) relatively increased towards the end of the outbreak (Fig. 2B).

Overall, respiratory symptoms (95\%), especially shortness of breath $(73 \%)$ and/or cough (68\%), were the most common symptoms reported, followed by fever $(81 \%)$, other constitutional symptoms (61\%), and gastrointestinal symptoms (39\%). For respiratory symptoms, patients reported more shortness of breath $(P<.01)$ but less sore throat $(P=.03)$ compared with HCWs. For other symptoms, patients reported more gastrointestinal symptoms $(P<.01)$ and dizziness or decreased level of consciousness $(P=.03)$ compared with HCWs. In all cases, respiratory symptoms (71\%) and/or fever (41\%) were by far the most frequently starting symptoms.

As shown in Table 3 , patients had clearly more comorbidity than HCWs in terms of its presence ( $99 \%$ vs $42 \%, P<.001$ ) or the number of comorbid diseases (5.6 [2.3] vs 2.0 [1.1], $P<.001)$. The most common diseases among patients were hypertension $(81 \%)$, diabetes $(78 \%)$, heart disease $(52 \%)$, chronic kidney disease (44\%), immunosuppressive disease $(41 \%)$, neurologic disease including stroke (28\%), respiratory disease $(25 \%)$, cancer $(22 \%)$, and dyslipidemia (20\%). All these diseases were significantly higher among patients than

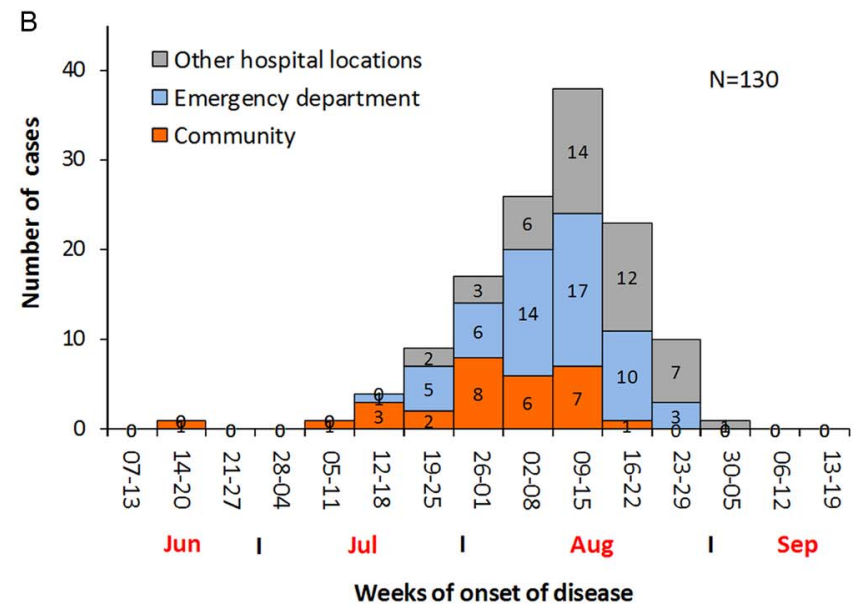

FIGURE 1. Epidemic curves describing the transmission of Middle East respiratory syndrome coronavirus infection during the outbreak at King Abdulaziz Medical City-Riyadh (mid-June to mid-September, 2015) including type of transmission (A) and probable exposure location (B). 
TA в LE 2. Clinical Symptoms of MERS-CoV Cases at KAMC-Riyadh (Mid-June to Mid-September, 2015)

\begin{tabular}{|c|c|c|c|c|}
\hline Variable & Patients $(\mathrm{N}=87)$ & HCWs $(\mathrm{N}=43)$ & Overall $(\mathrm{N}=130)$ & $P$ value \\
\hline \multicolumn{5}{|l|}{ Any symptoms } \\
\hline No & $3(3 \%)$ & $18(42 \%)$ & $21(16 \%)$ & \multirow[t]{2}{*}{$<.001$} \\
\hline Yes & $84(97 \%)$ & $25(58 \%)$ & $109(84 \%)$ & \\
\hline \multicolumn{5}{|l|}{ Number of all symptoms } \\
\hline Mean (SD) & $5.4(2.4)$ & $4.2(2.3)$ & $5.2(2.4)$ & .03 \\
\hline 1-3 symptoms & $18(21 \%)$ & $13(52 \%)$ & $31(28 \%)$ & \multirow[t]{3}{*}{.01} \\
\hline $4-6$ symptoms & $39(46 \%)$ & $9(36 \%)$ & $48(44 \%)$ & \\
\hline$\geq 7$ symptoms & $27(32 \%)$ & $3(12 \%)$ & $30(28 \%)$ & \\
\hline \multicolumn{5}{|l|}{ Number of respiratory symptoms } \\
\hline Mean $(\mathrm{SD})$ & $2.6(1.4)$ & $2.4(1.3)$ & $2.6(1.4)$ & .54 \\
\hline None & $4(5 \%)$ & $1(4 \%)$ & $5(5 \%)$ & \multirow[t]{3}{*}{.85} \\
\hline $1-2$ symptoms & $38(45 \%)$ & $13(52 \%)$ & $51(47 \%)$ & \\
\hline$\geq 3$ symptoms & $42(50 \%)$ & $11(44 \%)$ & $53(49 \%)$ & \\
\hline \multicolumn{5}{|l|}{ Symptoms } \\
\hline \multicolumn{5}{|l|}{ Respiratory } \\
\hline Any respiratory & $80(95 \%)$ & $24(96 \%)$ & $104(95 \%)$ & $>.99$ \\
\hline Cough & $57(68 \%)$ & $17(68 \%)$ & $74(68 \%)$ & .99 \\
\hline Shortness of breath & $67(80 \%)$ & $12(48 \%)$ & $79(73 \%)$ & $<.01$ \\
\hline Sore throat & $8(10 \%)$ & $7(28 \%)$ & $15(14 \%)$ & .03 \\
\hline Running nose & $6(7 \%)$ & $5(20 \%)$ & $11(10 \%)$ & .12 \\
\hline Chest pain & $29(35 \%)$ & $9(36 \%)$ & $38(35 \%)$ & .89 \\
\hline Hemoptysis & $7(8 \%)$ & $2(8 \%)$ & $9(8 \%)$ & \\
\hline Pneumonia & $24(29 \%)$ & $5(20 \%)$ & $29(27 \%)$ & .40 \\
\hline ARDS during the course & $23(27 \%)$ & $4(16 \%)$ & $27(25 \%)$ & .25 \\
\hline \multicolumn{5}{|l|}{ Constitutional } \\
\hline Any constitutional including fever & $80(95 \%)$ & $20(80 \%)$ & $100(92 \%)$ & .03 \\
\hline Any constitutional excluding fever & $52(62 \%)$ & $14(56 \%)$ & $66(61 \%)$ & .60 \\
\hline Fever & $71(85 \%)$ & $17(68 \%)$ & $88(81 \%)$ & .08 \\
\hline Headache & $12(14 \%)$ & $3(12 \%)$ & $15(14 \%)$ & $>.99$ \\
\hline Muscle aches & $21(25 \%)$ & $7(28 \%)$ & $28(26 \%)$ & .76 \\
\hline Fatigue/lethargy/weakness & $16(19 \%)$ & $3(12 \%)$ & $19(17 \%)$ & .55 \\
\hline Chills/rigors & $9(11 \%)$ & $3(12 \%)$ & $12(11 \%)$ & $>.99$ \\
\hline Dizziness or decrease LOC & $25(30 \%)$ & $2(8 \%)$ & $27(25 \%)$ & .03 \\
\hline Poor appetite & $12(14 \%)$ & $2(8 \%)$ & $14(13 \%)$ & .52 \\
\hline Rash & $4(5 \%)$ & $1(4 \%)$ & $5(5 \%)$ & $>.99$ \\
\hline Seizures & $0(0 \%)$ & $0(0 \%)$ & $0(0 \%)$ & NA \\
\hline \multicolumn{5}{|l|}{ Gastrointestinal } \\
\hline Any gastrointestinal & $39(46 \%)$ & $4(16 \%)$ & $43(39 \%)$ & $<.01$ \\
\hline Vomiting & $29(35 \%)$ & $3(12 \%)$ & $32(29 \%)$ & .03 \\
\hline Diarrhea & $16(19 \%)$ & $2(8 \%)$ & $18(17 \%)$ & .24 \\
\hline Abdominal pain/distention & $20(24 \%)$ & $2(8 \%)$ & $22(20 \%)$ & .08 \\
\hline \multicolumn{5}{|l|}{ Starting symptoms } \\
\hline Respiratory & $57(68 \%)$ & $20(80 \%)$ & $77(71 \%)$ & .24 \\
\hline Fever & $34(41 \%)$ & $11(44 \%)$ & $45(41 \%)$ & .75 \\
\hline Respiratory and fever & $17(20 \%)$ & $9(36 \%)$ & $26(24 \%)$ & .11 \\
\hline Other constitutional & $8(10 \%)$ & $3(12 \%)$ & $11(10 \%)$ & $>.99$ \\
\hline Gastrointestinal & $12(14 \%)$ & $1(4 \%)$ & $13(12 \%)$ & .29 \\
\hline
\end{tabular}

NOTE. The column heads represent the maximum number; the denominator was sometimes lower when data were missing. ARDS, acute respiratory distress syndrome; HCW, healthcare worker; KAMC, King Abdulaziz Medical City; LOC, level of consciousness; MERS-CoV, Middle East respiratory syndrome coronavirus.

HCWs with the exception of respiratory diseases, which were not significantly different between groups.

Of the 130 cases, 96 (74\%) were hospitalized and 34 (26\%) were home isolated (Table 4). Almost all patients (97\%) were hospitalized whereas the majority $(72 \%)$ of HCWs were home isolated. Among all 130 cases, 51 (39\%) died; these deaths represented $59 \%$ of patients and $61 \%$ of those who had been hospitalized. No deaths occurred among HCWs. The death 

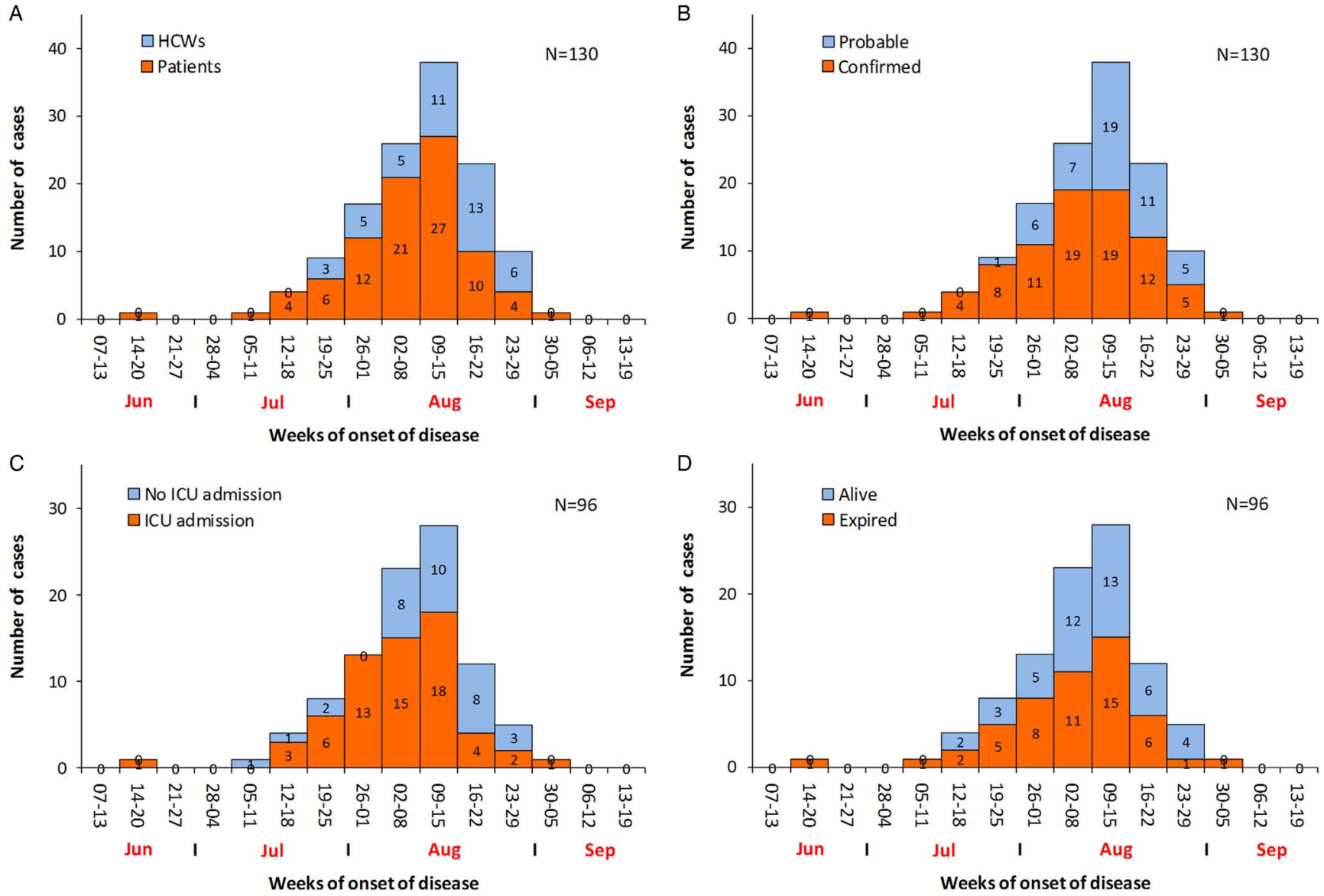

FIGURE 2. Epidemic curves describing the characteristics of Middle East respiratory syndrome coronavirus cases during the outbreak at King Abdulaziz Medical City-Riyadh (mid-June to mid-September, 2015) including type of case (A), diagnostic classification (B), need for intensive care unit admission (C), and discharge status (D).

rate was much higher in confirmed than probable cases $(56 \%$ vs $12 \%, P<.001)$. Among the 96 hospitalized cases, $63(66 \%)$ required ICU admission and $60(63 \%)$ required mechanical ventilation, with no difference between patients and HCWs. Additionally, there were no significant differences between patients and HCWs as regards hospital stay, ICU stay, or ventilation days. HCWs were suspected earlier after onset than patients $(1.5 \pm[2.7]$ vs $5.2 \pm[6.3]$ days, $P<.01)$. Moreover, the onset of symptoms occurred approximately 6 days after admission in HCWs but approximately 13 days before admission in patients. The change in the number of deaths and ICU admissions among hospitalized cases during the outbreak are shown in Figures 2C and 2D, respectively.

\section{I S C USSION}

We are reporting a large hospital outbreak affecting both patients and HCWs at a major tertiary care hospital in Riyadh. Although the attack rate among HCWs was reported to be lower than patients and family contacts, ${ }^{13} \mathrm{HCW}$ sepresented a significant proportion of all detected cases of MERS-CoV in the current study and elsewhere. One-third of the confirmed cases in the current outbreak were HCWs. This was similar to what was observed in the Jeddah outbreak, where $31 \%$ of the 255 confirmed cases were HCWs. ${ }^{14}$ Additionally, $40 \%$ of the 55 MERS-CoV cases reviewed in the United Arab Emirates were HCWs. ${ }^{15}$ However, it was considerably higher than seen in other outbreaks: $13 \%$ in the Eastern region outbreak and $14 \%$ in the Korean outbreak. ${ }^{5,8}$ Additionally, a review of the 99 cases detected after more than 5,000 screenings over a year in Saudi Arabia found that $19 \%$ were HCWs. ${ }^{13}$ One reason that may explain the high proportion of HCWs at the time of outbreak, including in the current study, is the active screening for patient contacts, mass screening of certain units that harbor patients, and proactive screening of anxious HCWs. Additionally, the higher than expected (from previous outbreaks) number of community cases of MERS-CoV may have amplified transmission and increased the risk of HCWs.

More than half of the healthcare-acquired infections in the current study were identified in the ED, a 150-bed multisection complex that served more than 800 visits per day during the outbreak. Factors that facilitated the spread of 
тав ве 3. Comorbidity of MERS-CoV cases at KAMC-Riyadh (mid-June to mid-September, 2015)

\begin{tabular}{|c|c|c|c|c|}
\hline Variable & Patients $(\mathrm{N}=87)$ & HCWs $(\mathrm{N}=43)$ & Overall $(\mathrm{N}=130)$ & $P$ value \\
\hline \multicolumn{5}{|l|}{ Any comorbid diseases } \\
\hline No & $1(1 \%)$ & $25(58 \%)$ & $26(20 \%)$ & \multirow[t]{2}{*}{$<.001$} \\
\hline Yes & $86(99 \%)$ & $18(42 \%)$ & $104(80 \%)$ & \\
\hline \multicolumn{5}{|l|}{ Number of comorbid diseases } \\
\hline Mean (SD) & $5.6(2.3)$ & $2.0(1.1)$ & $4.9(2.5)$ & $<.001$ \\
\hline 1-3 diseases & $16(19 \%)$ & $16(89 \%)$ & $32(31 \%)$ & \multirow[t]{3}{*}{$<.001$} \\
\hline 4-6 diseases & $41(48 \%)$ & $2(11 \%)$ & $43(41 \%)$ & \\
\hline$\geq 7$ diseases & $29(34 \%)$ & $0(0 \%)$ & $29(28 \%)$ & \\
\hline \multicolumn{5}{|l|}{ Respiratory comorbid diseases } \\
\hline Any respiratory disease & $22(25 \%)$ & $6(14 \%)$ & $28(22 \%)$ & .14 \\
\hline Asthma & $10(12 \%)$ & $3(7 \%)$ & $13(10 \%)$ & .54 \\
\hline Chronic obstructive pulmonary disease & $14(16 \%)$ & $3(7 \%)$ & $17(13 \%)$ & .15 \\
\hline Bronchiectasis & $3(3 \%)$ & $0(0 \%)$ & $3(2 \%)$ & .55 \\
\hline Tuberculosis & $5(6 \%)$ & $0(0 \%)$ & $5(4 \%)$ & .17 \\
\hline Allergic rhinitis & $1(1 \%)$ & $2(5 \%)$ & $3(2 \%)$ & .25 \\
\hline Other chronic respiratory disease & $6(7 \%)$ & $1(2 \%)$ & $7(5 \%)$ & .42 \\
\hline \multicolumn{5}{|l|}{ Other comorbid diseases } \\
\hline Hypertension & $70(81 \%)$ & $4(9 \%)$ & $74(57 \%)$ & $<.001$ \\
\hline Diabetes & $68(78 \%)$ & $3(7 \%)$ & $71(55 \%)$ & $<.001$ \\
\hline Heart disease & $45(52 \%)$ & $0(0 \%)$ & $45(35 \%)$ & $<.001$ \\
\hline Chronic kidney disease & $38(44 \%)$ & $0(0 \%)$ & $38(29 \%)$ & $<.001$ \\
\hline Hemodialysis & $13(15 \%)$ & $0(0 \%)$ & $13(10 \%)$ & .01 \\
\hline Immunosuppressive disease & $36(41 \%)$ & $0(0 \%)$ & $36(28 \%)$ & $<.001$ \\
\hline Neurologic disease & $24(28 \%)$ & $1(2 \%)$ & $25(19 \%)$ & $<.01$ \\
\hline Stroke & $21(24 \%)$ & $1(2 \%)$ & $22(17 \%)$ & $<.01$ \\
\hline Cancer & $19(22 \%)$ & $0(0 \%)$ & $19(15 \%)$ & $<.01$ \\
\hline Previous surgeries & $15(17 \%)$ & $3(7 \%)$ & $18(14 \%)$ & .11 \\
\hline Dyslipidemia & $17(20 \%)$ & $0(0 \%)$ & $17(13 \%)$ & $<.01$ \\
\hline Hypothyroidism & $12(14 \%)$ & $3(7 \%)$ & $15(12 \%)$ & .38 \\
\hline Other metabolic disease & $9(10 \%)$ & $2(5 \%)$ & $11(9 \%)$ & .34 \\
\hline Blood disease & $7(8 \%)$ & $3(7 \%)$ & $10(8 \%)$ & $>.99$ \\
\hline Urinary tract infection & $8(9 \%)$ & $2(5 \%)$ & $10(8 \%)$ & .50 \\
\hline Chronic liver disease & $7(8 \%)$ & $1(2 \%)$ & $8(6 \%)$ & .27 \\
\hline Benign prostatic hyperplasia & $7(8 \%)$ & $0(0 \%)$ & $7(5 \%)$ & .10 \\
\hline Psychiatric disease & $4(5 \%)$ & $0(0 \%)$ & $4(3 \%)$ & .30 \\
\hline Rheumatoid & $1(1 \%)$ & $0(0 \%)$ & $1(1 \%)$ & $>.99$ \\
\hline Other chronic disease & $17(20 \%)$ & $4(9 \%)$ & $21(16 \%)$ & .14 \\
\hline Bed bound & $8(9 \%)$ & $0(0 \%)$ & $8(6 \%)$ & .05 \\
\hline Pregnancy & $0(0 \%)$ & $1(2 \%)$ & $1(1 \%)$ & .33 \\
\hline
\end{tabular}

NOTE. The column heads represent the maximum number; the denominator was sometimes lower when data were missing. HCW, healthcare worker; KAMC, King Abdulaziz Medical City; MERS-CoV, Middle East respiratory syndrome coronavirus.

infection in and from the ED included the extreme overcrowding, uncontrolled patient movement, high visitor traffic, non-strict compliance with infection control recommendations, late recognition/isolation of some cases, and HCWs serving on both ED and other hospital locations. This was closely similar to the conditions believed to have contributed to the rapid spread of MERS-CoV in several hospitals in Saudi Arabia and South Korea. ${ }^{4,16}$ For example, combinations of several contributing factors, such as crowded ED environment, close and unprotected contacts with a symptomatic case, and possibly fomite transmission, have been suggested as causes in the Korean outbreak. ${ }^{16}$ Additionally, the ED received during the outbreak more than the expected number of community cases of MERS-CoV, which amplified ED transmission. This observation is also supported by the fact that there was an immediate decrease in new cases just after closure of the ED. Moreover, the commitment of KAMC-R to care for eligible patients regardless of the availability of beds and for noneligible cases in case of emergency, in addition to the lack of a divergence system, made this outbreak inevitable with difficult containment.

The current study reconfirmed significant differences between the MERS-CoV course and outcome among patients and HCWs at a healthcare setting. Obviously, HCWs were 
тав Le 4. Course and Outcome of MERS-CoV Cases at KAMC-Riyadh (Mid-June to Mid-September, 2015)

\begin{tabular}{|c|c|c|c|c|}
\hline Variable & Patients $(\mathrm{N}=87)$ & HCWs $(\mathrm{N}=43)$ & Overall $(\mathrm{N}=130)$ & $P$ value \\
\hline \multicolumn{5}{|l|}{ Isolation $(\mathrm{N}=130)$} \\
\hline Home isolation & $3(3 \%)$ & $31(72 \%)$ & $34(26 \%)$ & \multirow[t]{2}{*}{$<.001$} \\
\hline Hospitalization & $84(97 \%)$ & $12(28 \%)$ & $96(74 \%)$ & \\
\hline \multicolumn{5}{|l|}{ Outcome of isolation $(\mathrm{N}=130)$} \\
\hline Cleared home isolation & $3(3 \%)$ & $31(72 \%)$ & $34(26 \%)$ & \multirow[t]{3}{*}{$<.001$} \\
\hline Discharged & $33(38 \%)$ & $12(28 \%)$ & $45(35 \%)$ & \\
\hline Expired & $51(59 \%)$ & $0(0 \%)$ & $51(39 \%)$ & \\
\hline \multicolumn{5}{|l|}{ Outcome of hospitalization $(\mathrm{N}=96)$} \\
\hline Need ICU admission & $55(66 \%)$ & $8(67 \%)$ & $63(66 \%)$ & $>.99$ \\
\hline Need ventilation & $53(63 \%)$ & $7(58 \%)$ & $60(63 \%)$ & $>.99$ \\
\hline Expired & $51(61 \%)$ & $0(0 \%)$ & $51(53 \%)$ & $<.001$ \\
\hline Discharged & $33(39 \%)$ & $12(100 \%)$ & $45(47 \%)$ & $<.001$ \\
\hline \multicolumn{5}{|l|}{ Durations in hospital $(\mathrm{N}=96)^{\mathrm{a}}$} \\
\hline Total hospital days, mean $(\mathrm{SD})^{\mathrm{b}}$ & $29.0(23.8)$ & $19.9(14.4)$ & $28.0(23.1)$ & .25 \\
\hline$<2$ weeks & $24(29 \%)$ & $5(42 \%)$ & $29(30 \%)$ & \multirow[t]{3}{*}{.34} \\
\hline $2-4$ weeks & $33(39 \%)$ & $2(17 \%)$ & $35(37 \%)$ & \\
\hline$>4$ weeks & $27(32 \%)$ & $5(42 \%)$ & $32(33 \%)$ & \\
\hline Days between admission and onset, mean (SD) & $-13.4(80.4)$ & $5.9(2.9)$ & $-10.9(75.3)$ & $<.001$ \\
\hline Days to suspect after onset, mean (SD) & $5.2(6.3)$ & $1.5(2.7)$ & $4.4(5.9)$ & $<.01$ \\
\hline Hospital days before ICU admission, mean (SD) & $12.8(12.3)$ & $2.9(2.4)$ & $11.5(12.0)$ & $<.01$ \\
\hline ICU days, mean $(S D)^{\mathrm{b}}$ & $14.2(15.4)$ & $16.5(10.0)$ & $14.4(14.9)$ & .32 \\
\hline Ventilation days, mean $(\mathrm{SD})^{\mathrm{b}}$ & $11.1(11.9)$ & $14.6(6.7)$ & $11.4(11.6)$ & .13 \\
\hline Hospital days before death, mean (SD) & $34.9(99.1)$ & & $34.9(99.1)$ & NA \\
\hline ICU days before death, mean (SD) & $9.0(7.6)$ & & $9.0(7.6)$ & NA \\
\hline
\end{tabular}

NOTE. The column heads represent the maximum number; the denominator was sometimes lower when data were missing. HCW, healthcare worker; ICU, intensive care unit; KAMC, King Abdulaziz Medical City; MERS-CoV, Middle East respiratory syndrome coronavirus.

${ }^{\mathrm{a}}$ Mann-Whitney test.

${ }^{\mathrm{b}}$ Two HCW data outliers were removed from analysis.

younger, healthier, and with fewer comorbidities. Additionally, HCWs had a less severe version of the disease, as indicated by the higher frequency of asymptomatic cases and probable diagnosis. This may have reflected more intensive ascertainment rather than different disease behavior. For example, HCWs working in areas like ED and the dialysis unit were mass-screened to detect cases that otherwise would have been missed. Additionally, anxious HCWs were closely monitoring their symptoms and sought help frequently before they turned positive. This explanation was supported by the lack of major differences in the frequency of fever and respiratory symptoms among symptomatic cases of both patients and HCWs.

One of the most striking finding of the current study, yet previously reported, is the high mortality among patients and lack of such mortality among HCWs. Similarly, there was higher mortality among confirmed cases (mainly patients) than probable cases (mainly HCWs). This may reflect the younger age, better health, and earlier diagnosis among HCWs. This difference may reflect a detection bias that is typically seen in hospital outbreaks and may not be controlled except in a planned study design. The current $39 \%$ overall mortality and $59 \%$ patient mortality were similar to the Jeddah outbreak, where overall mortality was $36 \%$ and patient mortality was 59\%. ${ }^{14}$ Additionally, the overall mortality among accumulated cases in Saudi Arabia during the past 3 years was $43 \%{ }^{2}$ However, the current mortality numbers were much higher than reported in the Korean outbreak, where $36(19 \%)$ of the 186 died. $^{8}$ Interestingly, the mortality was $40 \%$ in the first half of the Korean outbreak before it significantly decreased at the end of outbreak. ${ }^{17}$ This was explained by the possibility of a better ascertainment rate and the infection of younger, healthier individuals in the second half of the outbreak. ${ }^{17}$ The mortality difference between the current and the Korean outbreaks may also reflect the higher comorbidity ( $80 \%$ vs $55 \%)$ and symptomatic disease $(81 \%$ vs $74 \%$ for fever and $68 \%$ vs $18 \%$ for cough) in the current outbreak compared with the Korean outbreak. ${ }^{8}$

The current outbreak reemphasizes the fact that MERS-CoV remains a major threat to advanced healthcare settings with a high possibility of hospital outbreaks. These outbreaks are costly in terms of lives, healthcare resources, and health service continuity. Early recognition of cases and rapid implementation of recommended infection control guidance is necessary to prevent and/or reduce the impact of healthcare-associated outbreaks, including MERS-CoV. However, most healthcare systems need to place drastic efforts into revamping national 
healthcare systems so that holistic infection control programs are adopted and not limited to the basics of hand hygiene and personal protective equipment. ${ }^{18}$

\section{ACKNOWLEDGMENTS}

We acknowledge the help of all members of the infection control departments at KAMC-R: Abrar Turkistani, Abubakar Fadlelmula, Ahmad Ibrahim, Ahmed Azzam, Ayham Al Badawi, Azzam Mohammed, Bassem Abukhzam, Bassema Shadfan, Dina Afurong, Eman Mazroa, Fatimah Abdulkarim, Francesca Bambilla, Gassem Anizi, Hisham Eid, Irma Trofeo, Joseph Tannous, Kassem Yassine, Kassem Zehri, Khalid Al Qahtani, Khawla Salman, Kholoud Ameer, Manal Ata, Marissa Alarcon, Moaied Matalkah, Mohammed Kleal, Mohammed Omair, Mohammed Qahtani, Nimfa Dagunton, Nouf Enizi, Nour Abdulmalek, Ohaila Slim, Rasha Al Ahmari, Tamer Abu Areshih, Teresa Portugal, Tina Bocobo, Waseem Khan, and Yahya Bakheet.

Financial support. None reported.

Potential conflicts of interest. All authors report no conflicts of interest relevant to this article.

Address correspondence to Hanan Balkhy, MD, Infection Prevention and Control Department, King Abdulaziz Medical City, Riyadh, PO Box 22490, Riyadh 11426 Kingdom of Saudi Arabia (balkhyh@ngha.med.sa).

\section{REFERENCES}

1. Zaki AM, van Boheemen S, Bestebroer TM, Osterhaus AD, Fouchier RA. Isolation of a novel coronavirus from a man with pneumonia in Saudi Arabia. N Engl J Med 2012;367:1814-1820.

2. Saudi Ministry of Health. MERS-CoV daily updates. Saudi Ministry of Health website. http://www.moh.gov.sa/en/CCC/ PressReleases/Pages/Statistics-2015-12-31-001.aspx. Published 2015. Accessed December 31, 2015.

3. World Health Organization (WHO). Middle East respiratory syndrome coronavirus (MERS-CoV)-Saudi Arabia. WHO website. http://www.who.int/csr/don/4-december-2015-mers-saudi-arabia/en/. Published December 4, 2015. Accessed December 31, 2015.

4. Al-Tawfiq JA, Perl TM. Middle East respiratory syndrome coronavirus in healthcare settings. Curr Opin Infect Dis 2015;28: 392-396.

5. Assiri A, McGeer A, Perl TM, et al. Hospital outbreak of Middle East respiratory syndrome coronavirus. N Engl J Med 2013;369: 407-416.

6. Drosten C, Muth D, Corman VM, et al. An observational, laboratory-based study of outbreaks of Middle East respiratory syndrome coronavirus in Jeddah and Riyadh, Kingdom of Saudi Arabia, 2014. Clin Infect Dis 2015;60:369-377.

7. Korean Society of Infectious Diseases. An unexpected outbreak of Middle East respiratory syndrome coronavirus infection in the Republic of Korea, 2015. Infect Chemother 2015;47:120-122.
8. Korea Centers for Disease Control and Prevention. Middle East respiratory syndrome coronavirus outbreak in the Republic of Korea, 2015. Osong Public Health Res Perspect 2015;6: 269-278.

9. Alameer K, Abukhzam B, Khan W, El-Saed A, Balkhy H. Middle East respiratory syndrome coronavirus (MERS-Cov) screening of exposed healthcare workers in a tertiary care hospital in Saudi Arabia. Antimicrob Resist Infect Control 2015;4: O57-O57.

10. Saudi Ministry of Health. Case definition and surveillance guidance - updated June 2015. Saudi Ministry of Health website. http://www.moh.gov.sa/en/CCC/Regulations/Case\%20Definition. pdf. Published June 2015. Accessed December 31, 2015.

11. Centers for Disease Control and Prevention (CDC). Interim infection prevention and control recommendations for hospitalized patients with Middle East respiratory syndrome coronavirus (MERS-CoV). CDC website. http://www.cdc.gov/coronavirus/ mers/infection-prevention-control.html\#infection-prevention. Updated December 8, 2015. Accessed December 31, 2015.

12. World Health Organization (WHO). Infection prevention and control of epidemic- and pandemic-prone acute respiratory diseases in health care. WHO website. http://apps.who.int/iris/ bitstream/10665/69707/1/WHO_CDS_EPR_2007.6_eng.pdf?ua=1. Published June 2007. Accessed December 31, 2015.

13. Memish ZA, Al-Tawfiq JA, Makhdoom HQ, et al. Screening for Middle East respiratory syndrome coronavirus infection in hospital patients and their healthcare worker and family contacts: a prospective descriptive study. Clin Microbiol Infect 2014;20: $469-474$.

14. Oboho IK, Tomczyk SM, Al-Asmari AM, et al. 2014 MERS-CoV outbreak in Jeddah-a link to health care facilities. $N$ Engl J Med 2015;372:846-854.

15. Das KM, Lee EY, Jawder SE, et al. Acute Middle East respiratory syndrome coronavirus: temporal lung changes observed on the chest radiographs of 55 patients. AJR Am J Roentgenol 2015;205: W267-S274.

16. World Health Organization (WHO). Middle East respiratory syndrome coronavirus (MERS-CoV): summary and risk assessment of current situation in the Republic of Korea and China, as of 19 June 2015. WHO website. http://www.who.int/entity/ emergencies/mers-cov/mers-cov-republic-of-korea-and-china-riskassessment-19-june-2015.pdf?ua=1. Published June 19, 2015. Accessed December 31, 2015.

17. Mizumoto K, Saitoh M, Chowell G, Miyamatsu Y, Nishiura H. Estimating the risk of Middle East respiratory syndrome (MERS) death during the course of the outbreak in the Republic of Korea, 2015. Intl J Infect Dis 2015;39:7-9.

18. Balkhy H. MERS CoV: a trigger for healthcare transformation. J Infect Public Health 2015;9:1-2. 